\title{
Hemostatic Radiotherapy for Gastric Cancer: Relationship Between Diffusion-Weighted Magnetic Resonance Images and Tumor Markers
}

Osamu Tanaka ( $\nabla$ c.bluered@gmail.com )

Asahi University: Asahi Daigaku https://orcid.org/0000-0002-7189-8407

Nobuaki Yagi

Asahi University: Asahi Daigaku

Masahiro Tawada

Asahi University: Asahi Daigaku

Takuya Taniguchi

Asahi University: Asahi Daigaku

Kousei Ono

Asahi University: Asahi Daigaku

Shuto Nakaya

Asahi University: Asahi Daigaku

Chiyoko Makita

Gifu University: Gifu Daigaku

Masayuki Matsuo

Gifu University: Gifu Daigaku

\section{Research Article}

Keywords: radiotherapy, gastric cancer, MRI, tumour marker

Posted Date: January 31st, 2022

DOI: https://doi.org/10.21203/rs.3.rs-1304780/v1

License: (c) (1) This work is licensed under a Creative Commons Attribution 4.0 International License.

Read Full License 


\section{Abstract}

Introduction: Pretreatment diagnosis by diffusion-weighted magnetic resonance imaging (DW-MRI) is useful to determine the effect of chemotherapy. Here we investigated the relationship among DW-MRI, endoscopy, and tumor markers.

Patients: Eight included patients underwent hemostatic radiotherapy (RT) for gastric cancer in this prospective study from 2019 to 2021 . The patients completed MRI, endoscopy, and blood tests before RT; MRI, endoscopy, and blood tests 1 month after RT; and MRI and blood tests 3 months after RT. Correlations between changes in apparent diffusion coefficient (ADC) derived from DW-MRI and the tumor marker carcinoembryonic antigen (CEA) were investigated.

Results: Univariate analysis of overall survival showed that sex and chemotherapy treatment were statistically significant factors. The CEA values before and 1 month after RT decreased significantly. There was no statistical difference between the CEA value 1 and 3 months after RT. The ADC value before and 1 month after RT increased significantly but not between 1 and 3 months after RT. Comparing the ratio of ADC before RT to 1 (or 3) month(s) after RT with that of CEA before RT to 1 (or 3) month(s) after $\mathrm{RT}$, we found an inverse relationship between the two ratios.

Conclusions: Therefore, changes in ADC and CEA are correlated. Additionally, 3 months after RT, the decrease in ADC appeared earlier than the decrease in CEA. ADC may indicate a biological change earlier than CEA, and the ratios of ADC and CEA may be important factors. These aspects warrant further confirmation in a larger sample population.

\section{Introduction}

Radiotherapy (RT) has a hemostatic effect on gastric cancer ${ }^{1-6}$. A response rate of $80 \%$ was obtained when patients were treated with RT at a dose of $20 \mathrm{~Gy}$ in five daily fractions ( $20 \mathrm{~Gy} / 5 \mathrm{fx}$ ). The treatment was judged to be successful if the hemoglobin level became stable 1 month after RT ${ }^{1}$. Tey et al. reported that the quality of life improved by $80 \%$ with RT at a dose of 36 Gy in 12 daily fractions ${ }^{2}$.

Endoscopy is the first choice for pretreatment diagnosis and the post-treatment evaluation of gastric cancer, but in recent years, there has been an increasing number of reports that diffusion-weighted magnetic resonance imaging (DW-MRI) is useful for preoperative staging ${ }^{7-9}$. The apparent diffusion coefficient (ADC) is a quantitative value ${ }^{10-15}$. Until now, endoscopic findings and tumor markers, such as carcinoembryonic antigen (CEA), obtained via blood tests, have been used to judge the therapeutic effects of treatment ${ }^{16,17}$. However, only the inner surface of the stomach can be observed with an endoscope.

Performing invasive examinations, such as endoscopy, on patients with advanced gastric cancer can be burdensome. Therefore, noninvasive diagnostic methods are necessary. No studies have investigated the relationship between the $A D C$ value as a diagnostic image and the CEA value as a blood diagnostic 
measure before and after RT, considering the endoscopic findings after hemostatic RT. This study aimed to clarify the relationship between $A D C$ and tumor markers (e.g., CEA) to obtain more accurate gastric cancer information noninvasively by combining data from blood and imaging tests.

\section{Materials And Methods \\ Eligibility}

Eight eligible patients completed the protocol out of 21 patients who received hemostatic RT for gastric cancer from 2019 to 2021 at XXXXX Hospital. All patients provided written informed consent. This clinical trial is registered in the UMIN clinical trial registry (XXXXX).

\section{Inclusion criteria for initial irradiation}

Patients with bleeding as identified by pathological and endoscopic examinations and those with hemoglobin levels of $\leq 8 \mathrm{~g} \mathrm{ml}^{-1}$ at the initial consultation were included. Continued bleeding resulting in anemia was identified by endoscopy. We performed whole-body contrast-enhanced computed tomography (CT) and gastric endoscopy; anemia caused by chronic disease was excluded via blood tests. Patients in whom a hemoglobin level of $\geq 8 \mathrm{~g} \mathrm{ml}^{-1}$ could not be maintained even after treatment with surgery, endoscopic hemostasis, or blood transfusion were excluded. Age, sex, tumor size, tumor location, initial hemoglobin level, blood transfusion, bypass surgery, chemotherapy, and Eastern Cooperative Oncology Group Performance status scores were not limited for the included patients.

\section{Exclusion criteria for initial irradiation}

Patients with a high risk of febrile neutropenia (neutrophil count $\leq 1000 / \mu \mathrm{l}$; platelet count $<30000 / \mathrm{mm}^{3}$ ), those with severe distant metastases (in the brain, lung, or liver) with a life expectancy of $<1$ month, and those with a medical history of anticoagulant use or RT to the abdomen were excluded.

\section{Image acquisition}

To obtain images without motion artifacts, scans were performed on an empty stomach to achieve reproducible results. Therefore, we injected butylscopolamine intramuscularly, obtained CT and MRI images, and planned the treatment. By doing so, we improved the reproducibility of each irradiation. CT (16-row multidetector computed tomography; Alexion, Toshiba Medical System; Otawara, Japan) and MRI (Achieva, Philips Medical Systems, Best, Netherlands) scans were performed to investigate the degree of tumor development. CT was performed with a slice thickness of $2 \mathrm{~mm}$, a field of view of $50 \times 50$ $\mathrm{cm}^{2}$, and settings of $150 \mathrm{mAs}$ and $120 \mathrm{kV}$. MRI parameters for T2-weighted imaging (T2WI) were as follows: fast spin-echo; repetition time (TR)/echo time (TE) in ms, 433/80; number of samples (signals) averaged (NSA), 1; Matrix, 256×204. DW-MRI parameters were as follows: gradient echo; TR/TE in ms, 1200/65; NSA, 5; Matrix, 80×142; B-value, 1000. We compared the intensity of the gastric wall in the DWMRI before RT (day 0), 30 days after RT (day 30; 1 month), and 90 days after RT (day 90; 3 months).

\section{Evaluation of images}


We observed the morphological characteristics of gastric cancer via T2WI and DW-MRI and measured a circle (diameter, $1-2 \mathrm{~cm}$ ) in the area containing the tumor as a region of interest. Measurements were taken before RT and 1 and 3 months after treatment. Endoscopy was performed before and 1 month after RT. The study protocol is shown in Figure 1.

\section{Radiotherapy planning}

We performed 20 Gy/5 fx of RT (Elekta Synergy System, Elekta Ltd, Crawley, UK). If the tumor's spread was not correctly measurable, we contoured the entire outer wall of the stomach. From the contoured line, the clinical target volume was contoured with a margin of $1 \mathrm{~cm}$ from the outer wall of the stomach, whereas the planned target volume was contoured with a margin of $1 \mathrm{~cm}$ from the clinical target volume.

\section{Extracted values}

The measured CEA value (unit: $\mathrm{ng} / \mathrm{ml}$ ).

The measured ADC value (unit: $10^{-3} \mathrm{~mm}^{2} / \mathrm{sec}$ ).

The ratio of the ADC values before and 1 (or 3) month(s) after RT.

The ratio of the CEA values before and 1 (or 3) month(s) after RT.

\section{Statistical analysis}

Statistical analysis was performed using univariate and multivariate analyses for each factor. Mauchly's sphericity test was performed on changes in the ADC and CEA values over time. Correlation between the $A D C$ and $C E A$ values was evaluated using a correlation matrix and simple regression analysis.

Association between each parameter and survival time was calculated using Cox regression proportional hazard analysis. A $p$-value of $<0.05$ was judged to be statistically significant (BellCurve for Excel Social Survey Research Information Co., Ltd. Tokyo, Japan).

\section{Results}

\section{Factors related to survival time}

Figure 2 shows endoscopic images before and 1 month after RT. Owing to the study design, the data was limited to those who survived up to 3 months and underwent all examinations. For overall survival, the univariate analysis showed that sex and the presence or absence of chemotherapy were significant factors (Table 1). However, only six males and two females were included herein. Male survival was 288 (range, 115-850) days, and female survival was 744 (541-947) days. At 1 and 3 months after RT, we evaluated the association between the ratio of CEA to ADC and survival time, but no strong correlation was observed. All factors except sex and chemotherapy (i.e., age, tumor size, tumor location, initial hemoglobin level, blood transfusion, and bypass surgery) were not found to be statistically significant in 
the Cox regression proportional hazard analysis. Endoscopy was performed before and 1 month after RT, and all patients showed a partial response.

Table 1

Univariate analysis of factors affecting overall survival

\begin{tabular}{|c|c|c|c|}
\hline Characteristics & \multicolumn{2}{|l|}{ Values } & p-values \\
\hline Sex & \multicolumn{2}{|c|}{ Male $=6$, female $=2$} & $<0.001$ \\
\hline Age (years) & \multicolumn{2}{|c|}{70.6 (range 66-81) } & 0.854 \\
\hline Performance status & $0-1$ & $2-3$ & 0.767 \\
\hline Chemotherapy & Yes $(n=5)$ & No $(n=3)$ & 0.001 \\
\hline Surgery & Yes $(n=1)$ & No $(n=7)$ & 0.076 \\
\hline Blood transfusion & Yes $(n=4)$ & No $(n=4)$ & 0.976 \\
\hline Initial response rate (EROTC) & $\begin{array}{l}\mathrm{CR}, \mathrm{PR}(\mathrm{n}= \\
8)\end{array}$ & $\begin{array}{l}\text { SD, } P D(n \\
=0)\end{array}$ & - \\
\hline Initial CEA value & \multicolumn{2}{|c|}{53.5 (range $2.1-147.2$ ) } & 0.554 \\
\hline Initial ADC value & \multicolumn{2}{|c|}{1318 (range 1063-1774) } & 0.376 \\
\hline $\begin{array}{l}\text { Ratio of CEA value between initial examination and } 1 \\
\text { month after radiotherapy }\end{array}$ & \multicolumn{2}{|c|}{0.87 (range $0.09-1.05$ ) } & 0.345 \\
\hline $\begin{array}{l}\text { Ratio of } A D C \text { value between initial examination and } 1 \\
\text { month after radiotherapy }\end{array}$ & \multicolumn{2}{|c|}{1.67 (range $1.06-2.32$ ) } & 0.398 \\
\hline $\begin{array}{l}\text { Ratio of CEA value between initial examination and } 3 \\
\text { months after radiotherapy }\end{array}$ & \multicolumn{2}{|c|}{1.12 (range $1.00-1.26$ ) } & 0.223 \\
\hline $\begin{array}{l}\text { Ratio of } A D C \text { value between initial examination and } 3 \\
\text { months after radiotherapy }\end{array}$ & \multicolumn{2}{|c|}{1.34 (range $0.09-1.65$ ) } & 0.465 \\
\hline
\end{tabular}

\section{Changes in carcinoembryonic antigen and apparent diffusion coefficient values}

The CEA values showed a statistically significant decrease from before RT to 1 month after RT, but no statistically significant change was observed between 1 month and 3 months after RT (Figure 3a). The $A D C$ values showed a statistically significant increase from before RT to 1 month after RT but decreased 3 months after RT compared with 1 month after RT (Figure 3b).

The ratio of $\mathrm{ADC}$ (before RT/1 month after RT) and the ratio of CEA (before RT/1 month after RT) showed a weak inverse correlation ( $r=-0.519$ ) (Figure 4a). Similarly, the ratio of ADC (before RT/3 months after 
RT) and the ratio of CEA (before RT/3 months after RT) showed a weak inverse correlation $(r=-0.499)$ (Figure 4b).

\section{Discussion}

It is not known how surgery on patients with advanced gastric cancer contributes to survival. In recent years, evidence for the effectiveness of hemostatic RT for gastric cancer has gradually increased, and it has become necessary to assess the therapeutic effect after irradiation $1,2,18$. In this study, we observed that changes in ADC and CEA are correlated. Additionally, ADC may indicate a biological change earlier than CEA, and the ratios of $A D C$ and CEA may be important factors predicting the therapeutic effects of treatment.

By improving the diagnostic accuracy of MRI for stage $\mathrm{T}$ and $\mathrm{N}$ gastric cancer, the findings are similar to those of endoscopic ultrasound and $\mathrm{CT}^{8-15}$. The usefulness of MRI for determining the effects of chemotherapy is also increasing. However, the methodology of hemostatic RT has not been wellestablished in pretreatment settings using butylscopolamine and CT/MRI including DW-MRI, and it is important to establish a noninvasive method for determining the effect of hemostatic RT.

DW-MRI (as measured by the ADC values) is useful for evaluating gastric cancer ${ }^{7-9,19}$. A weak correlation was found between an increase in the ratio of the ADC value and a decrease in the ratio of the CEA values (measured before and 1 or 3 months after RT). If the outlier in the top-left part of the plot in Figure $4 \mathrm{a}$ is excluded, the ratio of ADC (before RT/1 month after RT) and the ratio of CEA (before RT/1 month after RT) showed an inverse correlation $(r=-0.154)$. Similarly, if the outlier in Figure $4 \mathrm{~b}$ is excluded, the ratio of ADC (before RT/3 months after RT) and the ratio of CEA (before RT/3 months after RT) showed a strong inverse correlation $(r=-0.736)$. As our study only included eight cases, increasing the number of cases may clarify the roles of the ratios of ADC and CEA.

Recently, preoperative tumor, node, and metastases (TNM) stage diagnosis has been established using DW-MRI ${ }^{10,14,15}$. Additionally, DW-MRI is increasingly used to estimate the therapeutic effect of treatment, especially in chemotherapy.

For RT, the stomach shape must remain the same before, during, and after treatment, so RT was administered on an empty stomach. In addition to DW images, we also assessed T1-weighted, T2weigted, and fat saturation T2-weighted images. These are useful for observing the size of the gastric tumor (thickness of the stomach wall) and are required for qualitative measurement. DW-MRI is considered to be better for functional (biological) evaluation.

Previously, pretreatment stage diagnosis and the response to chemotherapy and RT had been measured by dilating the gastric content as much as possible with water to help locate the tumor by extending the stomach wall ${ }^{14,15}$. Stomach distension is certainly useful for determining tumor size, but when it comes to RT, it is necessary to reduce the size of the stomach. If this is not done, it would be difficult to irradiate 
in the same manner because of a large difference between the pretreatment diagnosis (on a stomach full of water) and treatment plan CT/MRI images (on an empty stomach). Therefore, pretreatment diagnosis and RT planning must be done using the same settings.

Due to the small number of cases in this study, no statistically significant relationship was found with survival time. In a previous study, hemostatic RT for gastric cancer was successful in 25 of 31 cases, but the median survival time was 91 (range, 46-299) days, and there were many cases of recurrence after 3 months ${ }^{1}$.

Considering that the ADC value decreased earlier than the CEA value raises the possibility that the ADC value may detect changes in the tumor earlier than the CEA value (Figure 3). An inverse correlation in the ratios of $A D C$ and CEA was observed in the subanalysis, which was strong if the outlier in the subanalysis was excluded. Nevertheless, future studies investigating the relationship between the ADC and CEA values on a larger sample population are warranted.

As this study only included eight cases that survived up to 3 months after treatment, the hemostatic effect naturally appeared after 1 month (hemoglobin and CEA values in the blood test), and all patients showed a partial response. Endoscopic findings showed that hemostasis was nearly achieved, with some tumors being scarred and some being ulcerated.

There is an inverse correlation between the ADC value and $\mathrm{T}$ and $\mathrm{N}$ staging ${ }^{11-15}$, which may be useful in predicting the response to neoadjuvant therapy. These values may potentially also serve as prognostic factors in assessing gastric cancer aggression.

When using ADC values in clinical practice, there is a lack of consensus on how to calculate and interpret them. They are calculated based on the region of interest, which can be drawn manually and be based on T2W or DW-MRI images with different $b$ values (800-1200). Some authors suggest using the maximum $A D C$ value, whereas others consider the average ADC value. This undermines the direct comparison of $A D C$ value results between studies and emphasizes the need for careful protocol standardization, image analysis, and measurement reproducibility between centers before implementation.

Three of the six men and both women in this study were able to receive chemotherapy. The fact that chemotherapy can be performed means that there is a hemostatic effect, and the general condition has improved, and it is considered a treatment bias when chemotherapy can be performed. Our results suggest that the presence of chemotherapy may contribute to survival. Two of the five patients were treated with nivolumab, and both survived for $>1$ year. The relationship between RT and immunotherapy has been reported by Herrera et al. ${ }^{20}$. Additionally, RT and immunotherapy are involved in hemostatic RT for gastric cancer, and hence, further investigation is needed.

In conclusion, the usefulness of DW-MRI has been reported mainly for solid tumors. Herein, we evaluated the usefulness of DW-MRI for gastric cancer using a tumor marker in RT settings. We showed that changes in the ADC values and changes in the CEA values are correlated using ratios (before/1 or 3 
months after RT). Additionally, 3 months after treatment, a decrease in ADC appeared earlier than a decrease in CEA. Our findings suggest that $A D C$ may represent biological changes earlier than CEA.

\section{Declarations}

Conflict of interest: None

Presentation at a conference: None

Clinical trial registration (Ethical approval): Yes

\section{References}

\section{XXXXX.}

2. Tey J, Zheng H, Soon YY, Leong CN, Koh WY, Lim K, et al. Palliative radiotherapy in symptomatic locally advanced gastric cancer: A phase II trial. Cancer Med. 2019;8:1447-58. https://doi.org/10.1002/cam4.2021.

3. $X X X X X$.

4. $X X X X X$.

5. $X X X X X$.

6. Lee YH, Lee JW, Jang HS. Palliative external beam radiotherapy for the treatment of tumor bleeding in inoperable advanced gastric cancer. BMC Cancer. 2017;17:541. https://doi.org/10.1186/s12885017-3508-x.

7. Digklia A, Wagner AD. Advanced gastric cancer: current treatment landscape and future perspectives. World J Gastroenterol. 2016;22:2403-14. https://doi.org/10.3748/wjg.v22.i8.2403.

8. Zhang Y, Yu J. The role of MRI in the diagnosis and treatment of gastric cancer. Diagn Interv Radiol. 2020;26:176-82. https://doi.org/10.5152/dir.2019.19375.

9. Borggreve AS, Goense L, Brenkman HJF, Mook S, Meijer GJ, Wessels FJ, et al. Imaging strategies in the management of gastric cancer: current role and future potential of MRI. Br J Radiol. 2019;92:20181044. https://doi.org/10.1259/bjr.20181044.

10. Liu S, Zhang Y, Chen L, Guan W, Guan Y, Ge Y, et al. Whole-lesion apparent diffusion coefficient histogram analysis: significance in T and N staging of gastric cancers. BMC Cancer. 2017;17:665. https://doi.org/10.1186/s12885-017-3622-9.

11. Onal Y, Samanci $C$. The role of diffusion-weighted imaging in patients with gastric wall thickening. Curr Med Imaging Rev. 2019;15:965-71. https://doi.org/10.2174/1573405614666181115120109.

12. Tang L, Wang XJ, Baba H, Giganti F. Gastric cancer and image-derived quantitative parameters: Part 2-a critical review of DCE-MRI and ${ }^{18}$ F-FDG PET/CT findings. Eur Radiol. 2020;30:247-60. https://doi.org/10.1007/s00330-019-06370-x. 
13. Soydan L, Demir AA, Torun M, Cikrikcioglu MA. Use of diffusion-weighted magnetic resonance imaging and apparent diffusion coefficient in gastric cancer staging. Curr Med Imaging. 2020;16:1278-89. https://doi.org/10.2174/1573405616666200218124926.

14. Liu S, Wang H, Guan W, Pan L, Zhou Z, Yu H, et al. Preoperative apparent diffusion coefficient value of gastric cancer by diffusion-weighted imaging: correlations with postoperative TNM staging. J Magn Reson Imaging. 2015;42:837-43. https://doi.org/10.1002/jmri.24841.

15. Joo I, Lee JM, Kim JH, Shin Cl, Han JK, Choi BI. Prospective comparison of 3T MRI with diffusionweighted imaging and MDCT for the preoperative TNM staging of gastric cancer. J Magn Reson Imaging. 2015;41:814-21. https://doi.org/10.1002/jmri.24586.

16. Cardoso R, Coburn N, Seevaratnam R, Sutradhar R, Lourenco LG, Mahar A, et al. A systematic review and meta-analysis of the utility of EUS for preoperative staging for gastric cancer. Gastric Cancer. 2012;15(Suppl 1):19-26. https://doi.org/10.1007/s10120-011-0115-4.

17. Saito T, Kurokawa Y, Takiguchi S, Miyazaki Y, Takahashi T, Yamasaki M, et al. Accuracy of multidetector-row $\mathrm{CT}$ in diagnosing lymph node metastasis in patients with gastric cancer. Eur Radiol. 2015;25:368-74. https://doi.org/10.1007/s00330-014-3373-9.

18. Saito T, Kosugi T, Nakamura N, Wada H, Tonari A, Ogawa $H$, et al. Treatment response after palliative radiotherapy for bleeding gastric cancer: a multicenter prospective observational study (JROSG 173). Gastric Cancer in press. https://doi.org/10.1007/s10120-021-01254-w.

19. XXXXX.

20. Herrera FG, Irving M, Kandalaft LE, Coukos G. Rational combinations of immunotherapy with radiotherapy in ovarian cancer. Lancet Oncol. 2019;20:e417-33. https://doi.org/10.1016/S14702045(19)30401-2.

\section{Figures}

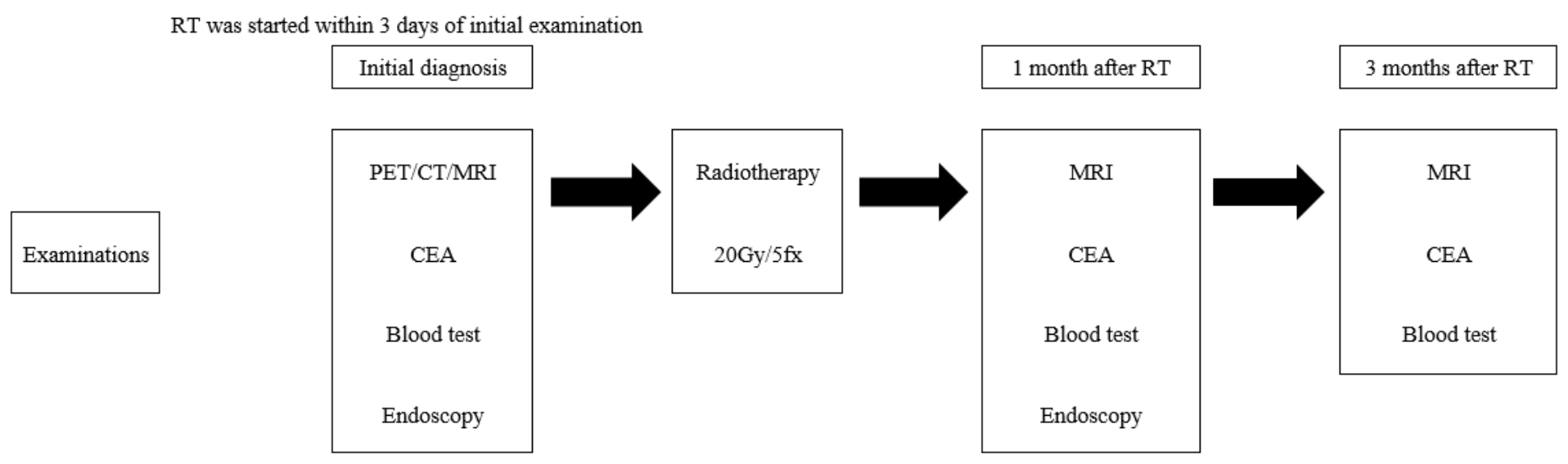

\section{Figure 1}

Study protocol 
RT Radiotherapy

PET ${ }^{18}$ F-fluorodeoxyglucose (FDG) positron emission tomography

CT Computed tomography

MRI Magnetic resonance diffusion weighted image

CEA Carcinoembryonic antigen

Blood test WBC, RBC, HGB, HCT, MCV, MCH, MCHC, PLT, NEUT, LYMPH, MONO, EOSINO, BASO, Ret, PT INR, APTT, FDP, AT3, CRP, TP, ALB, T-bil, D-bil, CHE, AST, ALT, ALP, g-GTP,LAP, LD, CK, AMY, Fe, TIBC, BUN, CRE, UA, Na, K, Cl, Ca, T-cho, TG, GLU, CRP, CEA, CA19-9

a

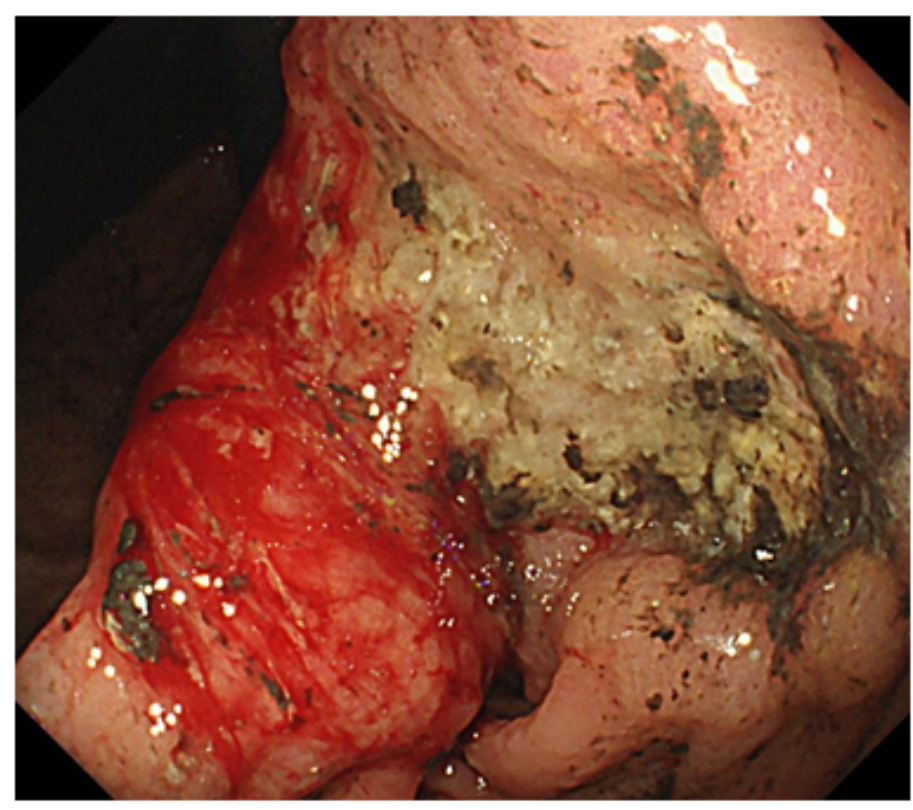

b

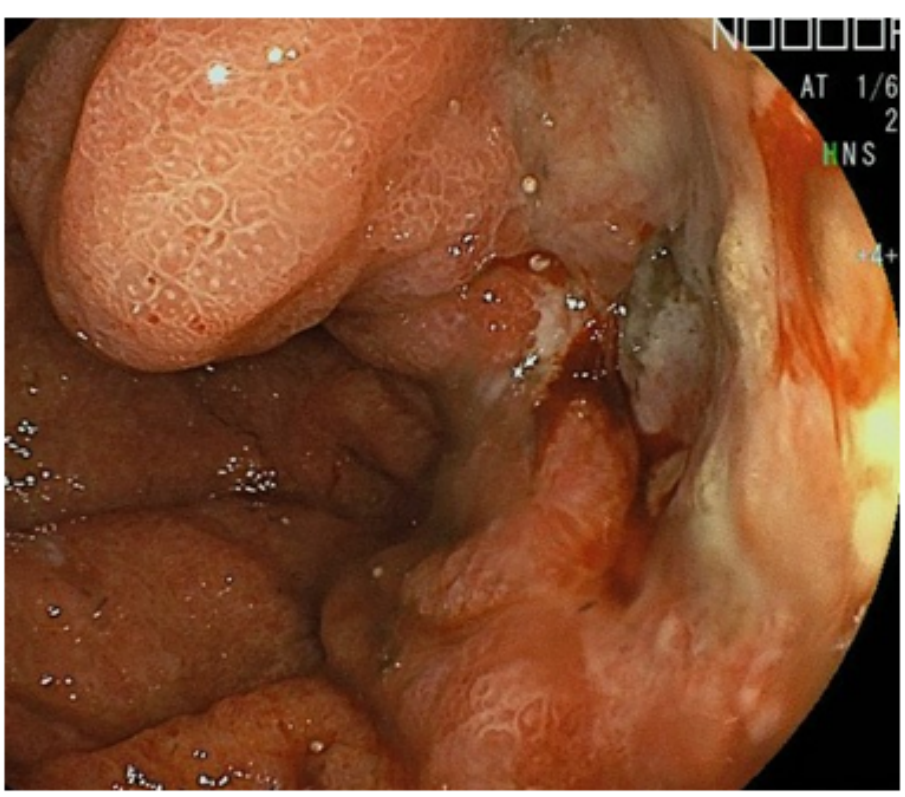

Figure 2

Endoscopic findings

2a) Before RT. Gastric cancer with ulceration and bleeding was observed from the angular incisure to the pylorus. Hemostasis treatment with an argon plasma laser for endoscopic treatment was unsuccessful.

2b) One month after RT. The tumor shrank considerably, with only a slight white moss and slight bleeding from a minor ulcer. 

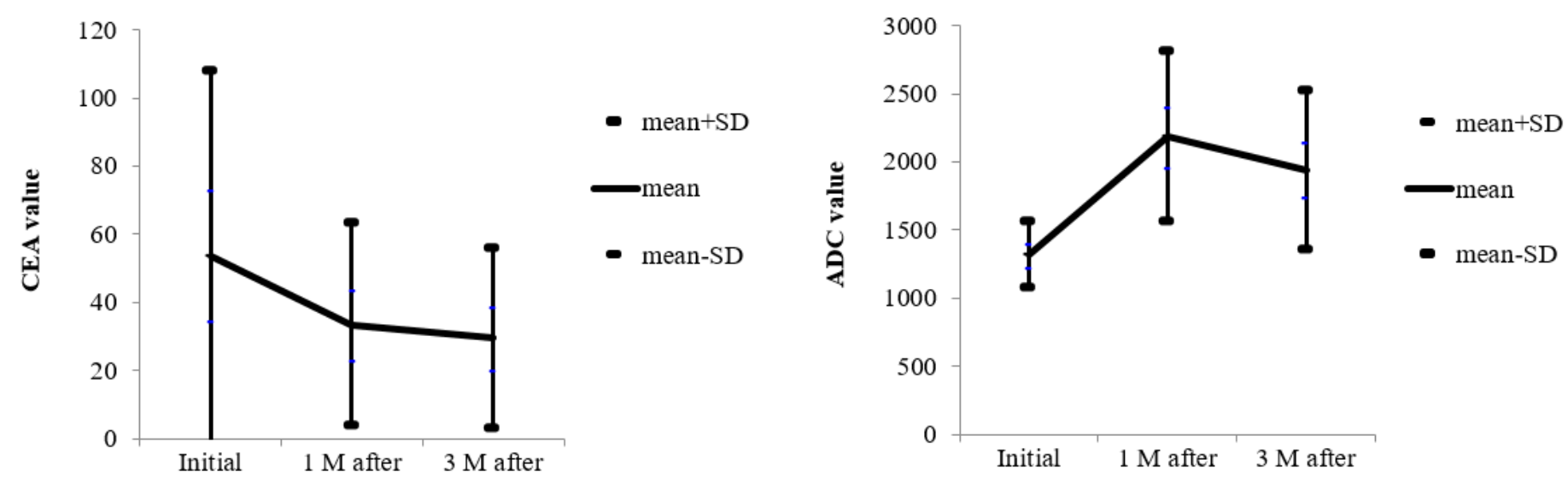

Figure 3

Changes in the measured CEA and ADC values

3a) Changes in the measured CEA values before RT and 1 and 3 months after RT.

3b) Changes in the measured ADC values before RT and 1 and 3 months after RT.

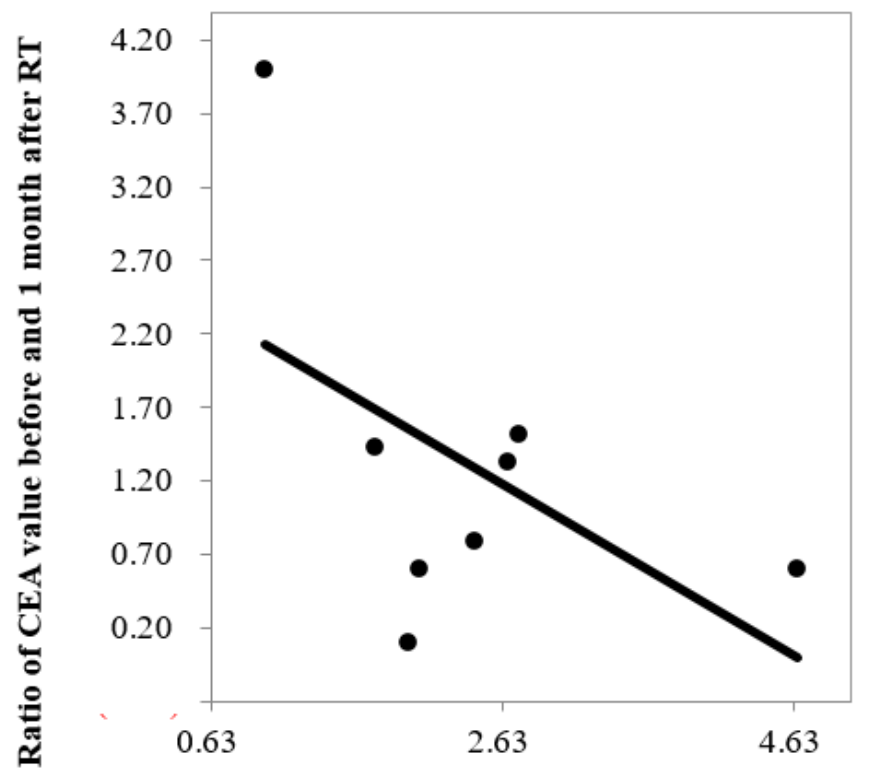

Ratio of ADC value before and 1 month after RT

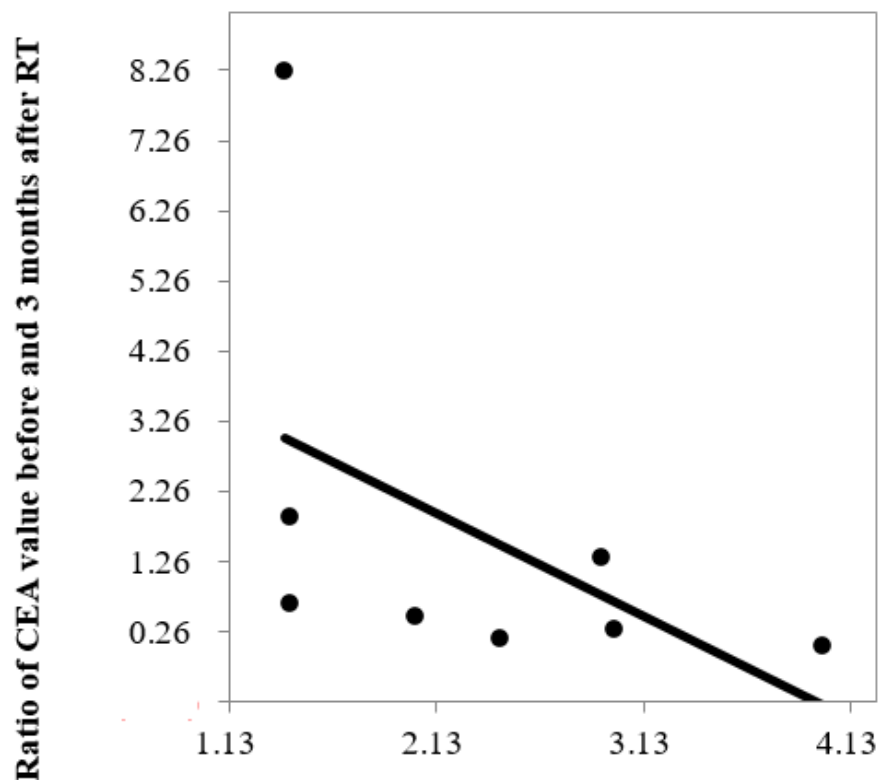

Ratio of ADC value before and 3 months after RT

Figure 4

Correlation between the ratios of $\mathrm{ADC}$ and CEA before and after RT

4a) Correlation between the ratio of ADC (before and 1 month after RT) to the ratio of CEA (before and 1 month after RT). 
4b) Correlation between the ratio of ADC (before and 3 months after RT) to the ratio of CEA (before and 3 months after RT). 